\title{
Error Exponents for Asymmetric Two-User Discrete Memoryless Source-Channel Systems*
}

\author{
Yangfan Zhong, Fady Alajaji and L. Lorne Campbell \\ Department of Mathematics and Statistics \\ Queen's University, Kingston, ON K7L 3N6, Canada \\ Email: \{yangfan,fady,campbll1 $\}$ mast.queensu.ca
}

\begin{abstract}
Consider transmitting two discrete memoryless correlated sources, consisting of a common and a private source, over a discrete memoryless multi-terminal channel with two transmitters and two receivers. At the transmitter side, the common source is observed by both encoders but the private source can only be accessed by one encoder. At the receiver side, both decoders need to reconstruct the common source, but only one decoder needs to reconstruct the private source. We hence refer to this system by the asymmetric 2-user source-channel system. In this work, we derive a universally achievable joint source-channel coding (JSCC) error exponent pair for the 2-user system by using a technique which generalizes Csiszár's method [3] for the pointto-point (single-user) discrete memoryless source-channel system. We next investigate the largest convergence rate of asymptotic exponential decay of the system (overall) probability of erroneous transmission, i.e., the system JSCC error exponent. We obtain lower and upper bounds for the exponent. As a consequence, we establish the JSCC theorem with single letter characterization.
\end{abstract}

\section{INTRODUCTION}

Recently, the study of the error exponent (reliability function) for point-to-point (single-user) source-channel systems has illustrated substantial superiority of joint source-channel coding (JSCC) over the traditional tandem coding (i.e., separate source and channel coding) approach (e.g., [3], [9]). It is of natural interest to study the JSCC error exponent for multi-terminal source-channel systems.

In this work we address the asymmetric 2-user sourcechannel system depicted in Fig. 1. Two discrete memoryless correlated source messages $(\mathbf{s}, \mathbf{l}) \in \mathcal{S}^{\tau n} \times \mathcal{L}^{\tau n}$ drawn from a joint distribution $Q_{S L}: \mathcal{S} \times \mathcal{L}$, consisting of a common source messages $\mathbf{s}$ and a private source message $\mathbf{l}$ of length $\tau n$, are transmitted over a discrete memoryless asymmetric communication channel described by $W_{Y Z \mid U X}: \mathcal{U} \times \mathcal{X} \rightarrow$ $\mathcal{Y} \times \mathcal{Z}$ with block codes of length $n$, where $\tau>0$ (measured in source symbol/channel use) is the overall transmission rate. The common source can be accessed by both encoders, but the private source can only be observed by one encoder (say, Encoder 1). In this set-up, the goal is to send the common information to both receivers, and send the private information to only one receiver (say, Decoder 1).

It is worthy to point out that the asymmetric 2-user system can be specialized to the following two classical asymmetric multi-terminal scenarios.

\footnotetext{
*This work was supported in part by NSERC of Canada
}

i.) The CS-AMAC system: If we remove Decoder 2 from Fig. 1, and let $|\mathcal{Z}|=1$, then the channel reduces to a multiple-access channel $W_{Y \mid U X}$, and the coding problem reduces to transmitting two correlated sources (CS) over an asymmetric multiple-access channel (AMAC) with one receiver.

ii.) The CS-ABC system: If we remove Encoder 2 from Fig. 1, and let $|\mathcal{U}|=1$, then the channel reduces to a broadcast channel $W_{Y Z \mid X}$, and the coding problem reduces to transmitting two $\mathrm{CS}$ over an asymmetric broadcast channel $(\mathrm{ABC})$ with one transmitter.

The sufficient and necessary condition for the reliable transmission of CS over the AMAC - i.e., the JSCC theorem for the CS-AMAC system - has been derived with single letter characterization in [5]. The capacity region of the $\mathrm{ABC}$ has been determined in [7], and the JSCC theorem for CS-ABC system with arbitrary transmission rate can also be analogously carried out (e.g., [6]). In this work, we study a refined version of the JSCC theorem for the general asymmetric 2-user system (depicted in Fig. 1), by investigating the achievable JSCC error exponent pair (for two receivers) as well as the system JSCC error exponent, i.e., the largest convergence rate of asymptotic exponential decay of the system (overall) probability of erroneous transmission. We also apply our results to the CSAMAC and CS-ABC systems.

We outline our results as follows. We first extend Csiszár's type packing lemma [3] from a single-letter (1-dimension) type setting to a joint (2-dimensional) type setting. By employing the joint type packing lemma, superposition encoders, and generalized maximum mutual information decoders, we establish a universally achievable error exponent pair for the two receivers (namely, the pair of exponents can be achieved by a sequence of source-channel codes independent of the statistics of the source and the channel); this generalizes Körner and Sgarro's exponent pair for ABC coding (with uniform message sets) [8]. We also employ a similar coding scheme to establish a lower bound for the system JSCC error exponent; see Theorem 1. Note that one consequence of our results is a sufficient condition (forward part) for the JSCC theorem. In addition, we use Fano's inequality to prove a necessary condition (converse part) which coincides with the sufficient condition, and hence completes the JSCC theorem (Theorem 2). Using an approach analogous to [3], we also 


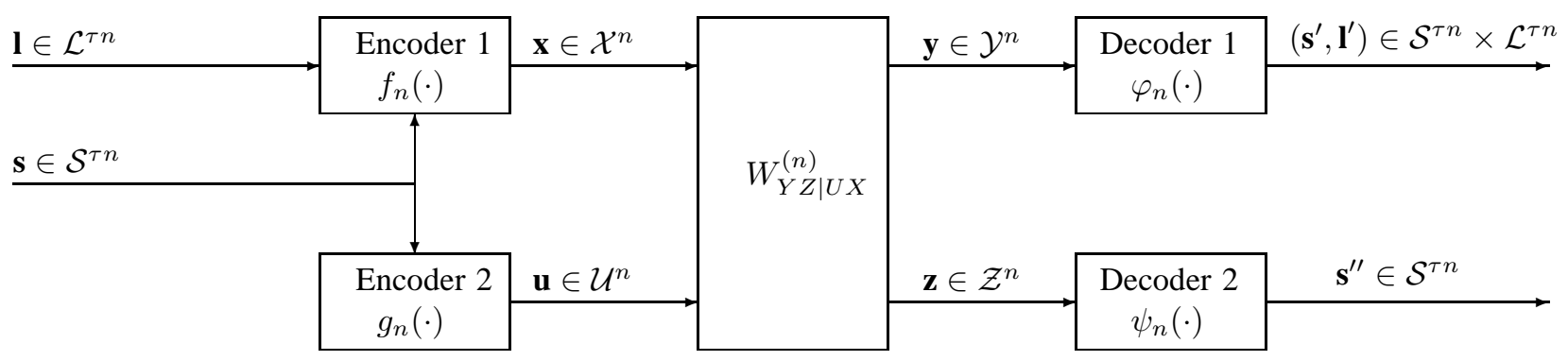

Fig. 1. Transmitting two CS over the asymmetric 2-user communication channel.

obtain an upper bound for the system JSCC error exponent (Theorem 3).

Due to limited space, we focus on the error exponents for the asymmetric 2-user system. In Section V, we briefly discuss applications to the CS-AMAC and the CS-ABC systems. All details and proofs are available in [10].

\section{NOTATION AND CONVENTIONS}

The following notations and conventions are adopted from [3], [4]. For any finite set (or alphabet) $\mathcal{X}$, the size of $\mathcal{X}$ is denoted by $|\mathcal{X}|$. The set of all probability distributions on $\mathcal{X}$ is denoted by $\mathcal{P}(\mathcal{X})$. We denote the type (the relative frequency of the components in a data sequence) of an $n$ length sequence $\mathbf{x} \in \mathcal{X}^{n}$ by $P_{\mathbf{x}} \in \mathcal{P}_{n}(\mathcal{X}) \subseteq \mathcal{P}(\mathcal{X})$, where $\mathcal{P}_{n}(\mathcal{X})$ is the collection of all types of sequences in $\mathcal{X}^{n}$. For any $P_{X} \in \mathcal{P}_{n}(\mathcal{X})$, the set of all $\mathbf{x} \in \mathcal{X}^{n}$ with type $P_{X}$ is denoted by $\mathbb{T}_{P_{X}}$, or simply by $\mathbb{T}_{X}$ if $P_{X}$ is understood. We also call $\mathbb{T}_{P_{X}}$ or $\mathbb{T}_{X}$ a type class. Similarly, the joint type of $n$-length sequences $\mathbf{x} \in \mathcal{X}^{n}$ and $\mathbf{y} \in \mathcal{Y}^{n}$ is denoted by $P_{\mathbf{x y}} \in \mathcal{P}_{n}(\mathcal{X} \times \mathcal{Y})$ and the set of all $\mathbf{x} \in \mathcal{X}^{n}$ and $\mathbf{y} \in \mathcal{Y}^{n}$ with joint type $P_{X Y} \in \mathcal{P}_{n}(\mathcal{X} \times \mathcal{Y})$ is denoted by $\mathbb{T}_{P_{X Y}}$, or simply by $\mathbb{T}_{X Y}$. For any finite sets $\mathcal{X}$ and $\mathcal{Y}$, the set of all conditional distributions $V_{Y \mid X}: \mathcal{X} \rightarrow \mathcal{Y}$ is denoted by $\mathcal{P}(\mathcal{Y} \mid \mathcal{X})$. The conditional type of $\mathbf{y} \in \mathcal{Y}^{n}$ given $\mathbf{x} \in \mathbb{T}_{P_{X}}$ is denoted by $P_{\mathbf{y} \mid \mathbf{x}} \in \mathcal{P}_{n}\left(\mathcal{Y} \mid P_{X}\right)$, where $\mathcal{P}_{n}\left(\mathcal{Y} \mid P_{X}\right)$ is the collection of all conditional distributions $V_{Y \mid X}$ which are conditional types of $\mathbf{y} \in \mathcal{Y}^{n}$ given an $\mathbf{x} \in \mathbb{T}_{P_{X}}$. For any conditional type $V_{Y \mid X} \in \mathcal{P}_{n}\left(\mathcal{Y} \mid P_{X}\right)$, the set of all $\mathbf{y} \in \mathcal{Y}^{n}$ for a given $\mathbf{x} \in \mathbb{T}_{P_{X}}$ satisfying $P_{\mathbf{y} \mid \mathbf{x}}=V_{Y \mid X}$ is denoted by $\mathbb{T}_{V_{Y \mid X}}(\mathbf{x})$, or simply by $\mathbb{T}_{Y \mid X}(\mathbf{x})$, which is also called a conditional type class with respect to $\mathbf{x}$. For finite sets $\mathcal{X}, \mathcal{Y}, \mathcal{Z}$ with joint distribution $P_{X Y Z} \in \mathcal{P}(\mathcal{X} \times \mathcal{Y} \times \mathcal{Z})$, we use $P_{X}, P_{X Y}, P_{Y Z \mid X}$, etc, to denote the corresponding marginal and conditional probabilities induced by $P_{X Y Z}$. Conversely, $P_{X} P_{Y Z \mid X}$ denotes a joint distribution on $\mathcal{X} \times \mathcal{Y} \times \mathcal{Z}$ with marginal distribution $P_{X}$ and conditional distribution $P_{Y Z \mid X}$. Note that for a given joint type $P_{X Y} \in \mathcal{P}_{n}(\mathcal{X} \times \mathcal{Y}), \mathbb{T}_{P_{Y \mid X}}(\mathbf{x})=\left\{\mathbf{y}:(\mathbf{x}, \mathbf{y}) \in \mathbb{T}_{P_{X Y}}\right\}$. Note also that

$\left\{P_{X} V_{Y \mid X}: P_{X} \in \mathcal{P}_{n}(\mathcal{X}), V_{Y \mid X} \in \mathcal{P}_{n}\left(\mathcal{Y} \mid P_{X}\right)\right\}=\mathcal{P}_{n}(\mathcal{X} \times \mathcal{Y})$
In addition, we denote

$$
\mathcal{P}_{n}(\mathcal{Y} \mid \mathcal{X}) \triangleq \bigcup_{P_{X} \in \mathcal{P}_{n}(\mathcal{X})} \mathcal{P}_{n}\left(\mathcal{Y} \mid P_{X}\right) \subseteq \mathcal{P}(\mathcal{Y} \mid \mathcal{X})
$$

To distinguish different distributions (or types) defined on the same alphabet, we use sub-subscript, say, $i, j$, in $P_{X_{i}}, P_{X_{i} Y_{j}}$, $\mathbb{T}_{X_{i} Y_{j}}$, and so on. For example, $\mathbb{T}_{X_{i} Y_{j}}$ is the type class of the joint type $P_{X_{i} Y_{j}} \in \mathcal{P}_{n}(\mathcal{X} \times \mathcal{Y})$. For any distribution $P_{X Y Z} \in$ $\mathcal{P}(\mathcal{X} \times \mathcal{Y} \times \mathcal{Z})$, we use $H_{P_{X Y Z}}(\cdot)$ and $I_{P_{X Y Z}}(\cdot ; \cdot)$ to denote the entropy and mutual information under $P_{X Y Z}$, respectively, or simply by $H(\cdot)$ and $I(\cdot ; \cdot)$ if $P_{X Y Z}$ is understood. $D\left(P_{X} \| Q_{X}\right)$ denotes the Kullback-Leibler divergence between distributions $P_{X}, Q_{X} \in \mathcal{P}(\mathcal{X}) . D\left(V_{Y \mid X} \| W_{Y \mid X} \mid P_{X}\right)$ denotes the Kullback-Leibler divergence between stochastic matrices (conditional distributions) $V_{Y \mid X}, W_{Y \mid X} \in \mathcal{P}(\mathcal{Y} \mid \mathcal{X})$ conditional on distribution $P_{X} \in \mathcal{P}(\mathcal{X})$. All logarithms and exponentials throughout this paper are in base 2 .

\section{A Joint Type PACKing Lemma}

We extend Csiszár's type packing lemma [3, Theorem. 5] from a (1-dimensional) single-letter type setting to a (2dimensional) joint type setting. This lemma plays a key role in deriving an exponentially achievable upper bound for the probability of erroneous transmission for the asymmetric 2user channel.

Lemma 1: (Joint Type Packing Lemma) Given finite sets $\mathcal{A}$ and $\mathcal{B}$, a sequence of positive integers $\left\{m_{n}\right\}$, and a sequence of positive integers $\left\{m_{i n}^{\prime}\right\}$ associated with every $i=1,2, \ldots, m_{n}$, for arbitrary (not necessarily distinct) types $P_{A_{i}} \in \mathcal{P}_{n}(\mathcal{A})$ and conditional types $P_{B_{j} \mid A_{i}} \in \mathcal{P}_{n}\left(\mathcal{B} \mid P_{A_{i}}\right)$, and positive integers $N_{i}$ and $M_{i j}, i=1,2, \ldots, m_{n}$ and $j=j(i)=1,2, \ldots, m_{i n}^{\prime}$ with $\frac{1}{n} \log _{2} N_{i}<H_{P_{A_{i}}}(A)-\delta$ and $\frac{1}{n} \log _{2} M_{i j}<H_{P_{A_{i}} P_{B_{j} \mid A_{i}}}(B \mid A)-\delta$, where

$$
\begin{aligned}
\delta \triangleq & \frac{2}{n}\left[|\mathcal{A}|^{2}|\mathcal{B}|^{2} \log _{2}(n+1)\right. \\
& \left.+\log _{2} m_{n}+\log _{2}\left(\max _{i} m_{i n}^{\prime}\right)+\log _{2} 12\right]
\end{aligned}
$$

there exist $m_{n}$ disjoint subsets $\Omega_{i}=\left\{\mathbf{a}_{p}^{(i)}\right\}_{p=1}^{N_{i}} \subseteq \mathbb{T}_{A_{i}} \triangleq$ $\mathbb{T}_{P_{A_{i}}}$ such that

$$
\left|\mathbb{T}_{V_{A^{\prime} \mid A}}\left(\mathbf{a}_{p}^{(i)}\right) \bigcap \Omega_{k}\right| \leq N_{k} 2^{-n\left[I_{P_{A_{i}} V_{A^{\prime} \mid A}}\left(A ; A^{\prime}\right)-\delta\right]},
$$


for every $i, k, p$ and $V_{A^{\prime} \mid A} \in \mathcal{P}_{n}(\mathcal{A} \mid \mathcal{A})$, with the exception of the case when both $i=k$ and $V_{A^{\prime} \mid A}$ is the conditional distribution such that $V_{A^{\prime} \mid A}\left(a^{\prime} \mid a\right)$ is 1 if $a^{\prime}=a$ and 0 otherwise; furthermore, for every $\mathbf{u}_{p}^{(i)} \in \Omega_{i}$ and every $i$, there exist $m_{i n}^{\prime}$ disjoint subsets $\Omega_{i j}\left(\mathbf{a}_{p}^{(i)}\right)=\left\{\left(\mathbf{a}_{p}^{(i)}, \mathbf{b}_{p, q}^{(j)}\right)\right\}_{q=1}^{M_{i j}}$ such that $\mathbf{b}_{p, q}^{(j)} \in \mathbb{T}_{B_{j} \mid A_{i}}\left(\mathbf{a}_{p}^{(i)}\right) \triangleq \mathbb{T}_{P_{B_{j} \mid A_{i}}}\left(\mathbf{a}_{p}^{(i)}\right)$ and

$$
\begin{aligned}
& \left|\mathbb{T}_{V_{A^{\prime} B^{\prime} \mid A B}}\left(\mathbf{a}_{p}^{(i)}, \mathbf{b}_{p, q}^{(j)}\right) \bigcap \bigcup_{p^{\prime}=1}^{N_{k}} \Omega_{k l}\left(\mathbf{a}_{p^{\prime}}^{(k)}\right)\right| \\
& \leq N_{k} M_{k l} 2^{-n\left[I_{P_{A_{i} B_{j}} V_{A^{\prime} B^{\prime} \mid A B}}\left(A, B ; A^{\prime}, B^{\prime}\right)-\delta\right],} \\
& \left|\mathbb{T}_{V_{A^{\prime} B^{\prime} \mid A B}}\left(\mathbf{a}_{p}^{(i)}, \mathbf{b}_{p, q}^{(j)}\right) \bigcap \bigcup_{p^{\prime}=1}^{N_{i}} \Omega_{i l}\left(\mathbf{a}_{p^{\prime}}^{(i)}\right)\right| \\
& \leq M_{i l} 2^{-n\left[I_{\left.P_{A_{i} B_{j}} V_{A^{\prime} B^{\prime} \mid A B}\left(B ; B^{\prime} \mid A\right)-\delta\right]}\right]}
\end{aligned}
$$

for any $i, j, k, l, p, q$ and $V_{A^{\prime} B^{\prime} \mid A B} \in \mathcal{P}_{n}(\mathcal{A} \times \mathcal{B} \mid \mathcal{A} \times \mathcal{B})$, with the exception of the case when both $i=k, j=l$ and $V_{A^{\prime} B^{\prime} \mid A B}$ is the conditional distribution such that $V_{A^{\prime} B^{\prime} \mid A B}\left(a^{\prime}, b^{\prime} \mid a, b\right)$ is 1 if $\left(a^{\prime}, b^{\prime}\right)=(a, b)$ and 0 otherwise.

We remark that the assertion of (1) is Csiszár's type packing lemma [3, Theorem 5] for a single-letter type setting. Roughly and intuitively, if $(\mathbf{a}, \mathbf{b})$ is a pair of transmitted codewords, then the possible sequences decoded as $(\mathbf{a}, \mathbf{b})$ can be seen as elements in the "sphere" $\mathbb{T}_{V_{A^{\prime} B^{\prime} \mid A B}}(\mathbf{a}, \mathbf{b})$ "centered" at $(\mathbf{a}, \mathbf{b})$ for some $V_{A^{\prime} B^{\prime} \mid A B}$. Eq. (2) in the packing lemma (similar to (1) and (3)) states that there exist disjoint sets $\Omega_{k l}=\bigcup_{p^{\prime}=1}^{N_{k}} \Omega_{k l}\left(\mathbf{a}_{p^{\prime}}^{(k)}\right)$ with bounded cardinalities such that the size of intersection between the sphere $\mathbb{T}_{V_{A^{\prime} B^{\prime} \mid A B}}(\mathbf{a}, \mathbf{b})$ for every $(\mathbf{a}, \mathbf{b}) \in \Omega_{i j}$ and every set $\Omega_{k l}$ is "exponentially small" compared with the size of each $\Omega_{k l}$. So the packing lemma can be used to prove the existence of good codes that have an exponentially small probability of error.

Note also that the above extended packing lemma is analogous to, but different from the one introduced by Körner and Sgarro in [8], which is used to prove a lower bound for the channel coding $\mathrm{ABC}$ exponent. Lemma 1 here is used for the JSCC problem.

\section{TRAnsmitting CS OVER the Asymmetric 2-User CHANNEL}

\section{A. System}

Let $\left\{W_{Y Z \mid U X}: \mathcal{U} \times \mathcal{X} \rightarrow \mathcal{Y} \times \mathcal{Z}\right\}$ be a 2-user discrete memoryless channel with finite input alphabet $\mathcal{U} \times \mathcal{X}$, finite output alphabet $\mathcal{Y} \times \mathcal{Z}$, and a transition distribution $W_{Y Z \mid U X}(y, z \mid u, x)$ such that the $n$-tuple transition probability is $W_{Y Z \mid U X}^{(n)}(\mathbf{y}, \mathbf{z} \mid \mathbf{u}, \mathbf{x})=\prod_{i=1}^{n} W_{Y Z \mid X}\left(y_{i}, z_{i} \mid u_{i}, x_{i}\right)$, where $u \in \mathcal{U}, x \in \mathcal{X}, y \in \mathcal{Y}, z \in \mathcal{Z}, \mathbf{u} \triangleq\left(u_{1}, \ldots, u_{n}\right) \in$ $\mathcal{U}^{n}, \mathbf{x} \triangleq\left(x_{1}, \ldots, x_{n}\right) \in \mathcal{X}^{n}, \mathbf{y} \triangleq\left(y_{1}, \ldots, y_{n}\right) \in \mathcal{Y}^{n}$, and $\mathbf{z} \triangleq\left(z_{1}, \ldots, z_{n}\right) \in \mathcal{Z}^{n}$. Denote the marginal transition distributions of $W_{Y Z \mid U X}$ at its $Y$-output (respectively $Z$ output) by $W_{Y \mid U X} \triangleq \sum_{Z} W_{Y Z \mid U X}$ (respectively $W_{Z \mid U X} \triangleq$
$\left.\sum_{Y} W_{Y Z \mid U X}\right)$. The marginal distributions of $W_{Y Z \mid U X}^{(n)}$ are denoted by $W_{Y \mid U X}^{(n)}$ and $W_{Z \mid U X}^{(n)}$, respectively.

Consider two discrete memoryless CS with a generic joint distribution $Q_{S L}(s, l)$ defined on the finite alphabet $\mathcal{S} \times$ $\mathcal{L}$ such that the $k$-tuple joint distribution is $Q_{S L}^{(k)}(\mathbf{s}, \mathbf{l})=$ $\prod_{i=1}^{k} Q_{S L}\left(s_{i}, l_{i}\right)$, where $(s, l) \in \mathcal{S} \times \mathcal{L}$, and $(\mathbf{s}, \mathbf{l}) \triangleq$ $\left(\left(s_{1}, l_{1}\right), \ldots,\left(s_{k}, l_{k}\right)\right) \in \mathcal{S}^{k} \times \mathcal{L}^{k}$. For each pair of source messages $(\mathbf{s}, \mathbf{l})$ drawn from the above joint distribution, we need to transmit the common message $\mathbf{s}$ over the channel $W_{Y Z \mid U X}$ to Receivers $Y$ and $Z$ and transmit the private message $\mathbf{l}$ only to Receiver $Y$. A joint source-channel (JSC) code with block length $n$ and transmission rate $\tau$ (source sumbol/channel use) for transmitting $Q_{S L}$ through $W_{Y Z \mid U X}$ is a quadruple of mappings, $\left(f_{n}, g_{n}, \varphi_{n}, \psi_{n}\right)$, where $f_{n}$ : $\mathcal{S}^{\tau n} \times \mathcal{L}^{\tau n} \rightarrow \mathcal{X}^{n}$ and $g_{n}: \mathcal{S}^{\tau n} \rightarrow \mathcal{U}^{n}$ are called encoders, and $\varphi_{n}: \mathcal{Y}^{n} \rightarrow \mathcal{S}^{\tau n} \times \mathcal{L}^{\tau n}$ and $\psi_{n}: \mathcal{Z}^{n} \rightarrow \mathcal{S}^{\tau n}$ are referred to as $Y$-decoder and $Z$-decoder, respectively; see Fig. 1.

The probabilities of $Y$ - and $Z$-error are given by

$$
\begin{aligned}
& P_{Y e}^{(n)}\left(Q_{S L}, W_{Y Z \mid U X}, \tau\right) \triangleq \operatorname{Pr}\left(\left\{\varphi_{n}(\mathbf{y}) \neq(\mathbf{s}, \mathbf{l})\right\}\right) \\
& =\sum_{\mathbf{s}, \mathbf{l}} Q_{S L}^{(\tau n)}(\mathbf{s}, \mathbf{l}) \sum_{\mathbf{y}: \varphi_{n}(\mathbf{y}) \neq(\mathbf{s}, \mathbf{l})} W_{Y \mid U X}^{(n)}(\mathbf{y} \mid \mathbf{u}, \mathbf{x})
\end{aligned}
$$

and

$$
\begin{aligned}
& P_{Z e}^{(n)}\left(Q_{S L}, W_{Y Z \mid U X}, \tau\right) \triangleq \operatorname{Pr}\left(\left\{\psi_{n}(\mathbf{z}) \neq \mathbf{s}\right\}\right) \\
& \quad=\sum_{\mathbf{s}, \mathbf{l}} Q_{S L}^{(\tau n)}(\mathbf{s}, \mathbf{l}) \sum_{\mathbf{z}: \psi_{n}(\mathbf{z}) \neq \mathbf{s}} W_{Z \mid U X}^{(n)}(\mathbf{z} \mid \mathbf{u}, \mathbf{x})
\end{aligned}
$$

where $\mathbf{x} \triangleq f_{n}(\mathbf{s}, \mathbf{l})$ and $\mathbf{u} \triangleq g_{n}(\mathbf{s})$ are the corresponding codewords of the source message pair $(\mathbf{s}, \mathbf{l})$ and the source message $\mathbf{s}$, and $\mathbf{y}$ and $\mathbf{z}$ are the received codewords at the Receivers $Y$ and $Z$, respectively. We say that the JSCC error exponent pair $\left(E_{A Y}, E_{A Z}\right)$ is achievable with respect to $\tau>0$ if there exists a sequence of JSC codes $\left(f_{n}, g_{n}, \varphi_{n}, \psi_{n}\right)$ with transmission rate $\tau$ such that the probabilities of $Y$-error and $Z$-error are simultaneously bounded by

$$
P_{i e}^{(n)}\left(Q_{S L}, W_{Y Z \mid U X}, \tau\right) \leq 2^{-n\left[E_{A i}-\delta\right]}, \quad i=Y, Z
$$

for $n$ sufficiently large and any $\delta>0$. As the point-to-point system, we denote the system (overall) probability of error by $P_{e}^{(n)}\left(Q_{S L}, W_{Y Z \mid U X}, \tau\right) \triangleq \operatorname{Pr}\left(\left\{\varphi_{n}(\mathbf{y}) \neq(\mathbf{s}, \mathbf{l})\right\} \cup\left\{\psi_{n}(\mathbf{z}) \neq \mathbf{s}\right\}\right)$, where $(\mathbf{s}, \mathbf{l})$ are drawn according to $Q_{S L}^{(\tau n)}$.

Definition 1: Given $Q_{S L}, W_{Y Z \mid U X}$ and $\tau>0$, the system JSCC error exponent $E_{J}\left(Q_{S L}, W_{Y Z \mid U X}, \tau\right)$ is defined as supremum of the set of all numbers $E$ for which there exists a sequence of JSC codes $\left(f_{n}, g_{n}, \varphi_{n}, \psi_{n}\right)$ with blocklength $n$ and transmission rate $\tau$ such that

$$
E \leq \liminf _{n \rightarrow \infty}-\frac{1}{n} \log _{2} P_{e}^{(n)}\left(Q_{S L}, W_{Y Z \mid U X}, \tau\right) .
$$

Since the system probability of error $P_{e}^{(n)}$ must be larger than $P_{Y e}^{(n)}$ and $P_{Z e}^{(n)}$ defined by (4) and (5), and is also upper bounded by the sum of the two, it follows that for any sequence of JSC codes $\left(f_{n}, g_{n}, \varphi_{n}, \psi_{n}\right)$

$$
\liminf _{n \rightarrow \infty}-\frac{1}{n} \log _{2} P_{e}^{(n)}=\liminf _{n \rightarrow \infty}-\frac{1}{n} \log _{2} \max \left(P_{Y e}^{(n)}, P_{Z e}^{(n)}\right) .
$$




\section{B. Superposition Encoding for Asymmetric 2-User Channels}

Given an asymmetric 2-user channel $W_{Y Z \mid U X}$, at the encoder side, we can artificially augment the channel input alphabet by introducing an auxiliary (arbitrary and finite) alphabet $\mathcal{T}$, and then look at the channel as a discrete memoryless channel $W_{Y Z \mid T U X}=W_{Y Z \mid U X}$ with marginal distributions $W_{Y \mid T U X}$ and $W_{Z \mid T U X}$ such that $W_{Y Z \mid T U X}(y, z \mid t, u, x)=$ $W_{Y Z \mid U X}(y, z \mid u, x)$ for any $t \in \mathcal{T}, u \in \mathcal{U}, x \in \mathcal{X}, y \in \mathcal{Y}$ and $z \in \mathcal{Z}$. In other words, we introduce a dummy $\operatorname{RV} T \in \mathcal{T}$ such that $T,(U, X)$, and $(Y, Z)$ form a Markov chain in this order, i.e., $T \rightarrow(U, X) \rightarrow(Y, Z)$.

The idea of superposition coding is described as follows. The encoder $g_{n}$ first maps the source message $\mathbf{s}$ to a pair of $n$-length sequences $(\mathbf{t}, \mathbf{u}) \in \mathcal{T}^{n} \times \mathcal{U}^{n}$ with a fixed type, say $P_{T U}$, and then sends the codeword $\mathbf{u}$ over the channel, i.e., $g_{n}(\mathbf{s})=\mathbf{u}$. The encoder $f_{n}$ first maps each pair $(\mathbf{s}, \mathbf{l})$ to a triple of sequences $(\mathbf{t}, \mathbf{u}, \mathbf{x}) \in \mathcal{T}^{n} \times \mathcal{U}^{n} \times \mathcal{X}^{n}$ such that $\mathbf{x} \in \mathbb{T}_{P_{X \mid T U}}(\mathbf{t}, \mathbf{u})$, then $f_{n}$ sends the codeword $\mathbf{x}$ over the channel, i.e., $f_{n}(\mathbf{s}, \mathbf{l})=\mathbf{x}$. In other words, $g_{n}$ and $f_{n}$ map $(\mathbf{s}, \mathbf{l})$ to a tuple of sequences $(\mathbf{t}, \mathbf{u}, \mathbf{x})$ with a joint type $P_{T U} P_{X \mid T U}$, although only $\mathbf{u}$ and $\mathbf{x}$ are sent to the channel, where $\mathbf{t}$ plays the role of a dummy codeword.

Since $W_{Y Z \mid T U X}^{(n)}(\mathbf{y}, \mathbf{z} \mid \mathbf{t}, \mathbf{u}, \mathbf{x})$ is equal to $W_{Y Z \mid U X}^{(n)}(\mathbf{y}, \mathbf{z} \mid \mathbf{u}, \mathbf{x})$ and is independent of $\mathbf{t}$, transmitting the codewords $(\mathbf{u}, \mathbf{x})$ through the channel $W_{Y Z \mid U X}$ can be viewed as transmitting the codewords $(\mathbf{t}, \mathbf{u}, \mathbf{x})$ over the augmented channel $W_{Y Z \mid T U X}$. Here, the common outputs of $g_{n}$ and $f_{n},(\mathbf{t}, \mathbf{u})$ 's, are called auxiliary cloud centers according to the traditional superposition coding notion [2], which convey the information of the common message $\mathbf{s}$, and the codewords $\mathbf{x}$ 's corresponding to the same $(\mathbf{t}, \mathbf{u})$ are called satellite codewords of $(\mathbf{t}, \mathbf{u})$, which contain both the common and private information. At the decoding stage, Receiver $Z$ only needs to figure out which cloud $(\mathbf{t}, \mathbf{u})$ was transmitted, and Receiver $Y$ needs to estimate not only the cloud but also the satellite codeword $\mathbf{x}$. We employ superposition encoding to derive the achievable error exponent pair and the lower bound of system JSCC error exponent in Section IV-C.

\section{Achievable Exponents and a Lower Bound for $E_{J}$}

Given arbitrary and finite alphabet $\mathcal{T}$, for any joint distribution $P_{T U X} \in \mathcal{P}(\mathcal{T} \times \mathcal{U} \times \mathcal{X})$ and every $R_{1}>0, R_{2}>0$, define

$$
\begin{aligned}
& E_{Y}\left(R_{1}, R_{2}, W_{Y \mid T U X}, P_{T U X}\right) \\
& \triangleq \min _{V_{Y \mid T U X}}\left[D\left(V_{Y \mid T U X} \| W_{Y \mid T U X} \mid P_{T U X}\right)\right. \\
& +\min \left(\left|I_{P_{T U X} V_{Y \mid T U X}}(T, U, X ; Y)-\left(R_{1}+R_{2}\right)\right|^{+}\right. \\
& \left.\left.\quad\left|I_{P_{T U X} V_{Y \mid T U X}}(X ; Y \mid T, U)-R_{2}\right|^{+}\right)\right]
\end{aligned}
$$

and

$$
\begin{aligned}
& E_{Z}\left(R_{1}, R_{2}, W_{Z \mid T U X}, P_{T U X}\right) \\
& \triangleq \min _{V_{Z \mid T U X}}\left[D\left(V_{Z \mid T U X} \| W_{Z \mid T U X} \mid P_{T U X}\right)\right. \\
& \left.\quad+\left|I_{P_{T U X} V_{Z \mid T U X}}(T, U ; Z)-R_{1}\right|^{+}\right]
\end{aligned}
$$

where $|x|^{+}=\max (0, x)$, and the outer minimum in (8) (respectively (9)) is taken over all conditional distributions on $\mathcal{P}(\mathcal{Y} \mid \mathcal{T} \times \mathcal{U} \times \mathcal{X})$ (respectively $\mathcal{P}(\mathcal{Z} \mid \mathcal{T} \times \mathcal{U} \times \mathcal{X})$ ). Using Lemma 1 and employing superposition encoders and generalized maximum mutual information decoders at the two receivers, we can prove the following achievable bounds.

Theorem 1: Given arbitrary and finite alphabet $\mathcal{T}$, for any $\widetilde{P}_{T U X} \in \mathcal{P}(\mathcal{T} \times \mathcal{U} \times \mathcal{X})$, the following exponent pair is universally achievable,

$$
\begin{array}{r}
E_{J Y}\left(Q_{S L}, W_{Y Z \mid T U X}, \widetilde{P}_{T U X}, \tau\right) \triangleq \min _{P_{S L}}\left[\tau D\left(P_{S L} \| Q_{S L}\right)\right. \\
\left.+E_{Y}\left(\tau H_{P}(S), \tau H_{P}(L \mid S), W_{Y \mid T U X}, \widetilde{P}_{T U X}\right)\right],(10)
\end{array}
$$

and

$$
\begin{array}{r}
E_{J Z}\left(Q_{S L}, W_{Y Z \mid T U X}, \widetilde{P}_{T U X}, \tau\right) \triangleq \min _{P_{S L}}\left[\tau D\left(P_{S L} \| Q_{S L}\right)\right. \\
\left.\quad+E_{Z}\left(\tau H_{P}(S), \tau H_{P}(L \mid S), W_{Z \mid T U X}, \widetilde{P}_{T U X}\right)\right],(11)
\end{array}
$$

where $W_{Y \mid T U X}$ and $W_{Z \mid T U X}$ are marginal distributions of $W_{Y Z \mid T U X}$, which is the augmented conditional distribution from $W_{Y Z \mid U X}$. Furthermore, given $Q_{S L}, W_{Y Z \mid U X}$, and $\tau$, the system JSCC error exponent satisfies

$$
\begin{gathered}
E_{J}\left(Q_{S L}, W_{Y Z \mid U X}, \tau\right) \geq \min _{P_{S L}}\left[\tau D\left(P_{S L} \| Q_{S L}\right)\right. \\
\left.+E_{r}\left(\tau H_{P}(S), \tau H_{P}(L \mid S), W_{Y Z \mid U X}\right)\right]
\end{gathered}
$$

where

$$
\begin{aligned}
& E_{r}\left(R_{1}, R_{2}, W_{Y Z \mid U X}\right) \\
& \quad \triangleq \sup _{\mathcal{T}} \max _{P_{T U X}} E_{r}\left(R_{1}, R_{2}, W_{Y Z \mid T U X}, P_{T U X}\right),
\end{aligned}
$$

where the supremum is taken over all finite alphabets $\mathcal{T}$, and the maximum is taken over all the joint distributions on $\mathcal{P}(\mathcal{T} \times$ $\mathcal{U} \times \mathcal{X})$ and $E_{r}\left(R_{1}, R_{2}, W_{Y Z \mid T U X}, P_{T U X}\right)$ is given by

$$
\begin{array}{r}
\min \left\{E_{Y}\left(R_{1}, R_{2}, W_{Y \mid T U X}, P_{T U X}\right),\right. \\
\left.E_{Z}\left(R_{1}, R_{2}, W_{Z \mid T U X}, P_{T U X}\right)\right\},
\end{array}
$$

where $E_{Y}$ and $E_{Z}$ are given by (8) and (9), respectively.

We remark that (10) and (11) can be achieved by a sequence of codes without the knowledge of $Q_{S L}$ and $W_{Y Z \mid U X}$, but the lower bound (12) is achieved by a sequence of codes that needs to know the statistics of the channel.

By examining the positivity of the lower bound to $E_{J}$, we obtain a sufficient condition for reliable transmissibility for the asymmetric 2-user system. For the sake of completeness, we also prove a converse by using Fano's inequality, and hence establish the JSCC theorem for this system. Given $W_{Y Z \mid U X}$, define

$$
\mathcal{R}\left(W_{Y Z \mid U X}\right) \triangleq \bigcup_{\mathcal{T}} \bigcup_{P_{T U X} \in \mathcal{P}(\mathcal{T} \times \mathcal{U} \times \mathcal{X})} \mathcal{R}\left(W_{Y Z \mid T U X}, P_{T U X}\right)
$$

where the first union is taken over all discrete alphabets $\mathcal{T}$ with $|\mathcal{T}| \leq|\mathcal{U}||\mathcal{X}|+2$, and $\mathcal{R}\left(W_{Y Z \mid T U X}, P_{T U X}\right)$ is defined by the following set

$$
\left\{\begin{array}{ll}
R_{1}+R_{2}<I(T, U, X ; Y) \\
\left(R_{1}, R_{2}\right): \quad R_{1}<I(T, U ; Z) \\
R_{2}<I(X ; Y \mid T, U)
\end{array}\right\},
$$


where the mutual informations are taken under the joint distribution $P_{T U X Y Z}=P_{T U X} W_{Y Z \mid U X}$.

Theorem 2: (JSCC Theorem) Given $Q_{S L}, W_{Y Z \mid U X}$ and $\tau>0$, the following statements hold.

(1) The sources can be transmitted over the channel with $P_{e}^{(n)} \rightarrow 0$ as $n \rightarrow \infty$ if $\left(\tau H_{Q}(S), \tau H_{Q}(L \mid S)\right) \in$ $\mathcal{R}\left(W_{Y Z \mid U X}\right)$

(2) Conversely, if the sources can be transmitted over the channel with an arbitrarily small probability of error $P_{e}^{(n)}$ as $n \rightarrow \infty$, then $\left(\tau H_{Q}(S), \tau H_{Q}(L \mid S)\right) \in \mathcal{R}\left(W_{Y Z \mid U X}\right)$ with $<$ replaced by $\leq$ in $\mathcal{R}\left(W_{Y Z \mid U X}\right)$.

\section{The Upper Bound to $E_{J}$}

In [3], Csiszár also established an upper bound for the JSCC error exponent for the point-to-point discrete memoryless source-channel system in terms of the source and channel error exponents by a simple type counting argument. He shows that the JSCC error exponent is always less than the infimum of the sum of the source and channel error exponent, even though the channel error exponent is only partially known for high rates. This conceptual bound cannot currently be computed as the channel error exponent is not yet fully known for all achievable coding rates, but it directly implies that any upper bound for the channel error exponent yields a corresponding upper bound for the JSCC error exponent. For the asymmetric 2-user channel, it can be shown by using a similar approach based on the method of types that the following is true.

Theorem 3: Given $Q_{S L}, W_{Y Z \mid U X}$, and $\tau$, the system JSCC error exponent satisfies

$$
\begin{aligned}
& E_{J}\left(Q_{S L}, W_{Y Z \mid U X}, \tau\right) \leq \inf _{P_{S L}}\left[\tau D\left(P_{S L} \| Q_{S L}\right)\right. \\
& \left.\quad+E\left(\tau H_{P}(S), \tau H_{P}(L \mid S), W_{Y Z \mid U X}\right)\right],
\end{aligned}
$$

where $E\left(\cdot, \cdot, W_{Y Z \mid U X}\right)$ is the corresponding channel coding error exponent for the asymmetric 2-user channel (refer to [10] for the formal definition).

\section{Applications to CS-AMAC and CS-ABC Systems}

We note briefly that our results of the previous section can be directly applied to the CS-AMAC system (by setting $|\mathcal{Z}|=1$ and removing $\left.\psi_{n}(\cdot)\right)$ and the CS-ABC system (by setting $|\mathcal{U}|=1$ and removing $g_{n}(\cdot)$ ). Here we remark that, when applying Theorem 3 to the CS-AMAC system, we can use the upper bound of the channel error exponent $E\left(\cdot, \cdot, W_{Y Z \mid U X}\right)$ in (14) for the general multiple-access channel (with one common message set and two private message sets) derived in [1] and obtain a looser but (in some cases) computable upper bound to $E_{J}$. In the following we give an example to show that for a class of CS-AMAC pairs the lower bound (12) and the upper bound on (14) coincide for a wide range of source-channel conditions. Similarly, Theorems 1-3 apply to the CS-ABC system. The readers may refer to [10] for the full details.

Example: (Binary CS-AMAC System) Consider two binary CS $Q_{S L}$ with distribution $Q_{S L}(S=0, L=0)=\frac{2(1-q)}{3}$,
$Q_{S L}(S=1, L=0)=\frac{q}{2}$, and $Q_{S L}(S=0, L=1)=\frac{q}{2}$, where $0<q<1 / 2$, and a binary AMAC $W_{Y \mid U X}$ with binary additive noise $P_{F}(F=1)=\epsilon(0<\epsilon<1 / 2)$ and output described by $Y_{i}=U_{i} \oplus X_{i} \oplus F_{i}(\bmod 2), \mathcal{Y}=\mathcal{U}=\mathcal{X}=\mathcal{F}=$ $\{0,1\}$, and $F_{i}$ is independent of $X_{i}$ and $U_{i}, i=1,2, \ldots, n$. In Fig. 2, we plot the lower and upper bounds for the JSCC error exponent $E_{J}$ for different $(q, \epsilon)$ pairs with transmission rate $\tau=0.25,0.35$. As illustrated, the upper and lower bounds coincide (this can also be proved) for many $(q, \epsilon)$ pairs (e.g., when $\tau=0.25, q=0.1, \epsilon \geq 0.0205$ and when $\tau=0.35, q=$ $0.1, \epsilon \geq 0.0056$ ), and hence exactly determine the exponent.

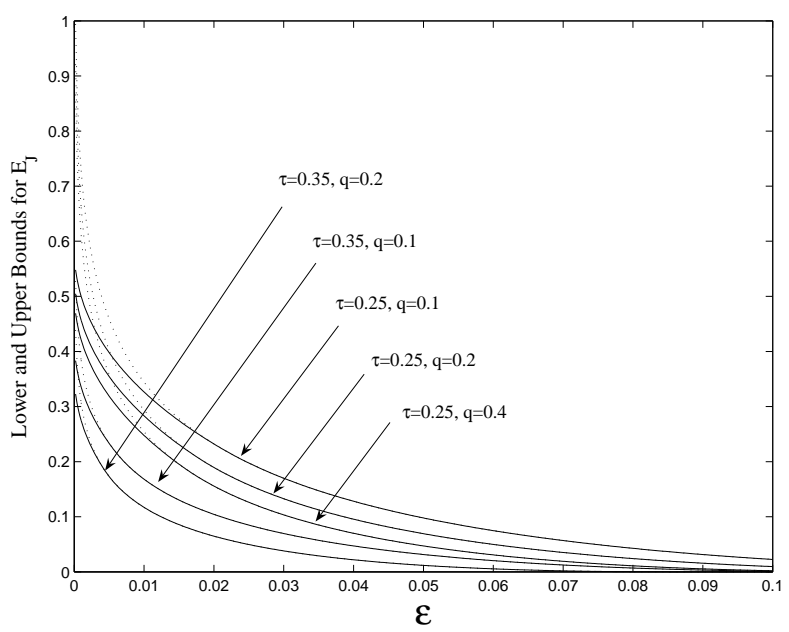

Fig. 2. The lower bound (solid line) and the upper bound (dash line) for the system JSCC error exponent for transmitting binary CS over the binary AMAC with binary additive noise.

\section{REFERENCES}

[1] E. A. Arutyunyan (Haroutunian), "Lower bound for the error probability of multiple-access channels," Probl. Pered. Inform., vol. 11, pp. 23-36, April-June 1975.

[2] P. P. Bergmans, "Random coding theorem for broadcast channels with degraded components," IEEE Trans. Inform. Theory, vol. 19, pp. 197206, Mar. 1973.

[3] I. Csiszár, "Joint source-channel error exponent," Probl. Contr. Inform. Theory, vol. 9, pp. 315-328, 1980.

[4] I. Csiszár and J. Körner, Information Theory: Coding Theorems for Discrete Memoryless Systems. New York: Academic, 1981.

[5] K. De Bruyn, V. V. Prelov, and E. Van Der Meulen, "Reliable transmission of two correlated sources over an asymmetric multiple-acess channel," IEEE Trans. Inform. Theory, vol. 33, pp. 716-718, Sep. 1987.

[6] T. S. Han and M. H. M. Costa, "Broadcast channels with arbitrary correlated sources," IEEE Trans. Inform. Theory, vol. 33, no. 5, pp. 641650, Sep. 1987.

[7] J. Körner and K. Marton, "Genreal broadcast channels with degraded message sets," IEEE Trans. Inform. Theory, vol. 23, pp. 60-64, Jan. 1977.

[8] J. Körner and A. Sgarro, "Universally attainable error exponents for broadcast channels with degraded message sets," IEEE Trans. Inform. Theory, vol. 26, pp. 670-679, Nov. 1980.

[9] Y. Zhong, F. Alajaji, and L. L. Campbell, "On the joint source-channel coding error exponent for discrete memoryless systems," IEEE Trans. Inform. Theory, vol. 52, no. 4, pp. 1450-1468, April 2006.

[10] Y. Zhong, F. Alajaji, and L. L. Campbell, "Coding error exponents for asymmetric two-user discrete memoryless source-channel systems," Mathematics and Engineering Technical Report, Dept. Math. and Stats., Queen's Univ., Kingston, ON, Canada, March 2007. Available [Online] at http://markov.mast.queensu.ca/TR/cs-ac-exp-TR07.pdf 